\title{
Fuel supplier selection for large scale UK bioenergy schemes
}

\author{
James A. Scott ${ }^{1, *}$, William Ho ${ }^{1}$, Prasanta Kumar Dey ${ }^{1}$ \\ ${ }^{1}$ Aston University, Birmingham, UK \\ * Corresponding author. Tel: +44 1212043210 Fax: +44 121204 3326, E-mail: scottja1@aston.ac.uk
}

\begin{abstract}
This article presents a potential method to assist developers of future bioenergy schemes when selecting from available suppliers of biomass materials. The method aims to allow tacit requirements made on biomass suppliers to be considered at the design stage of new developments. The method used is a combination of the Analytical Hierarchy Process and the Quality Function Deployment methods (AHP-QFD). The output of the method is a ranking and relative weighting of the available suppliers which could be used to improve optimization algorithms such as linear and goal programming. The paper is at a conceptual stage and no results have been obtained. The aim is to use the AHP-QFD method to bridge the gap between treatment of explicit and tacit requirements of bioenergy schemes; allowing decision makers to identify the most successful supply strategy available.
\end{abstract}

Keywords: AHP, QFD, Bioenergy, supplier selection

\section{Introduction}

The UK Bioenergy industry is expected to undergo significant growth over the coming decade as utilities and government aim to reach renewable energy targets by 2020. This expected growth is due to increasing installations of biomass heating, biofuel production for transport, biochemical for oil substitution, combined heat and power production and centralized electricity generation from biomass. For the sector to succeed the rapid development of demand for biomass resources must be matched by a sustainable supply. The various different bioenergy conversion processes that can be used to supply this range of lower carbon products brings a diverse set of material suppliers to the attention of project developers and procurement managers.

These fuels are likely to arise from a wide variety of sources and will have greatly differing properties and characteristics such as varying moisture or energy content. Additionally there are likely to be both positive and negative impacts associated with deciding to use a particular supplier. These impacts are less well defined when compared to the explicitly expressed measures of material properties and cost. The more tacit properties of a biomass fuel could include labor hours, $\mathrm{CO}_{2}$ emissions, air water and noise pollution, job creation, waste diverted from landfill, price fluctuation and reliability of supply are all examples of impacts a bioenergy scheme may have upon wider society and the environment. The challenge for the procurement manager is to decide which sources of materials to select and how much of each material to purchase from each supplier, thus creating a supply strategy.

Most conversion plants will have some technical parameters to which the input feedstock blend should comply with; these parameters define the desired fuel specification required of the blend. The problem of which blend to use lends itself well to goal programming techniques as the relationships are linear and the optimal blend can be expected to be a mixture of materials from different sources. Several methods have previously been successfully applied to the optimization of the bioenergy supply chain under various contexts. For instance for a multi-fuel problem and maximizing some objective function; energy efficiency, net $\mathrm{CO}_{2}$ emissions, or labor hours [1]. The output of such decision models is to 
give recommendations on the optimal location and capacity of new bioenergy plants, or to suggest an optimal supply or logistics strategy.

Previous research on the tacit impacts of a bioenergy system also exists. Often these tacit requirements are described in the context of sustainability metrics described as social and environmental impacts. Key sustainability constraints for UK bioenergy schemes have been identified as greenhouse gas savings, land availability, air quality, and problems associated with facility siting [2]. In a study on decision making for sustainable energy schemes some assessment criteria were identified and categorized as ecological, social and economic and included factors such as employment rate, land competition and supply security [3]. Indeed the study of sustainability regarding biomass grown for energy use has attracted a great amount of academic and public attention over the past decade.

The understanding of these two sides of the supplier selection problem is fairly robust considering the relative immaturity of the sector and the small number of commercially operating schemes. There is a gap in the treatment of the supplier selection problem however and more widely in the design of the biomass supply chain. The existing studies are unable to fully combine the optimization algorithms used for explicit aspects with knowledge of tacit requirements made of suppliers. This work presents a possible method to bridge this gap between the treatment of tacit and explicit requirements. The output of the work will be a structured process for developers to follow which will allow a score to be generated for each supplier given the extent to which that supplier meets the requirements of the development and any identified critical stakeholders.

The method behind the proposed framework is the combined AHP-QFD supplier selection method [4]. The hypothesis is that by selecting biomass suppliers using the AHP-QFD method a combination of suppliers can be selected to provide a supply which more effectively meets the needs of the conversion facility whilst remaining within the feasible region of cost and technical requirements.

This approach allows developers to move their procurement strategy beyond the model of transaction cost theories which are not suitable for the non-commodity market faced by the bioenergy industry sector at present. Building relationships between suppliers and conversion facilities will allow suppliers to better understand and meet the requirements of the conversion facility. Increasing the degree to which requirements are satisfied and maintaining a competitive cost for the fuel compared to other supply options. This is expected to be a better model for all parties than either the transaction cost model or the vertically integrated supply chain model.

\section{Methodology}

The AHP-QFD method has been applied in several previous cases for the selection problem under various multi-criteria conditions. The AHP-QFD method has been frequently applied in the manufacturing sector to select engineering projects [5] and more commonly to the area of product design $[6,7]$ that QFD was initially developed for. Elsewhere the method has also been applied to selecting budgets, teams and facility locations within logistics problems.

Current practice is for developers to select supply blends based on a mixture of experience and market knowledge as well as the price of each supply. The AHP-QFD method will be used to generate a recommended supply blend for a given scheme with a supply blend designed using current practice. The two recommended supply blends will then be compared with one another to determine the extent to which key tacit requirements are met by each approach. 


\subsection{The QFD Method}

Quality Function Deployment (QFD) allows the requirements of a customer to be mapped against the characteristics of a product. The House of Quality technique is closely associated with QFD and allows for this translation or mapping to be done systematically. The method uses one or more interrelationship matrices to relate the properties of the product to the requirements of the customer. The customer requirements are given a weighting related to its importance to the customer. The person or team of people completing the matrix is required to judge to what extent each requirement is met by each product characteristic. The output of the process is an importance score for each of the product characteristics.

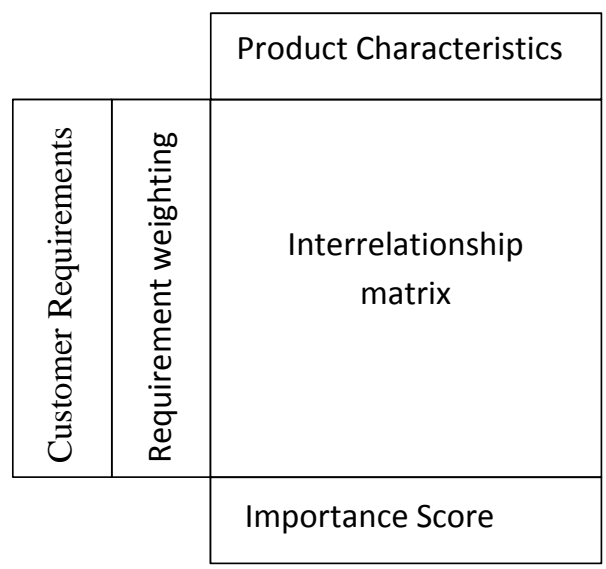

Fig. 1. A typical construction of a product HoQ.

The great advantage of the HoQ and QFD method is that each product characteristic is given a relative importance related to the degree to which that characteristic meets the customers' requirements. The weakness of the method is that the assigned importance is heavily reliant upon accurate completion of the interrelationship matrix. Any inconsistency or inaccuracy in this part of the HoQ process will lead to misleading final importance scores. Obtaining accurate weightings for the customer requirements is also important. This weakness is overcome when the Analytical Hierarchy Process (AHP) is applied.

\subsection{The AHP-QFD Method}

This section describes the steps used to determine the relationship weightings between the list of product characteristics and the customer requirements thus completing the interrelationship matrix. The following steps describe the AHP for use in a House of Quality.

Step 1: Construct a comparison matrix A with a customer requirement for each row and a product characteristic for each column.

$$
A=\left[\begin{array}{cccc}
a_{11} & a_{12} & \cdots & a_{1 n} \\
a_{12} & a_{22} & \cdots & a_{2 n} \\
\vdots & \vdots & \ddots & \vdots \\
a_{n 1} & a_{n 2} & \cdots & a_{n n}
\end{array}\right],
$$

Where $n$ is the number of elements in the top array (Product Characteristics), and $a_{i j}$ is the comparison of element $i$ to element $j$ using a 9-point scale shown in table 1. 
Step 2: AHP Synthesization

Divide each entry of the matrix A by the column total. This creates a normalized comparison matrix $A$ '.

$$
A^{\prime}=\left[\begin{array}{ccc}
\frac{a_{11}}{\sum_{i \in R} a_{i 1}} & \cdots & \frac{a_{1 n}}{\sum_{i \in R} a_{i n}} \\
\frac{a_{n 1}}{\sum_{i \in R} a_{i 1}} & \cdots & \frac{a_{n n}}{\sum_{i \in R} a_{i n}}
\end{array}\right],
$$

Where $R$ is a set of customer requirements $R=\{1,2, \ldots, n\}$.

Step 3: Create a column vector $C$ from the averages of each row of matrix $A$ '.

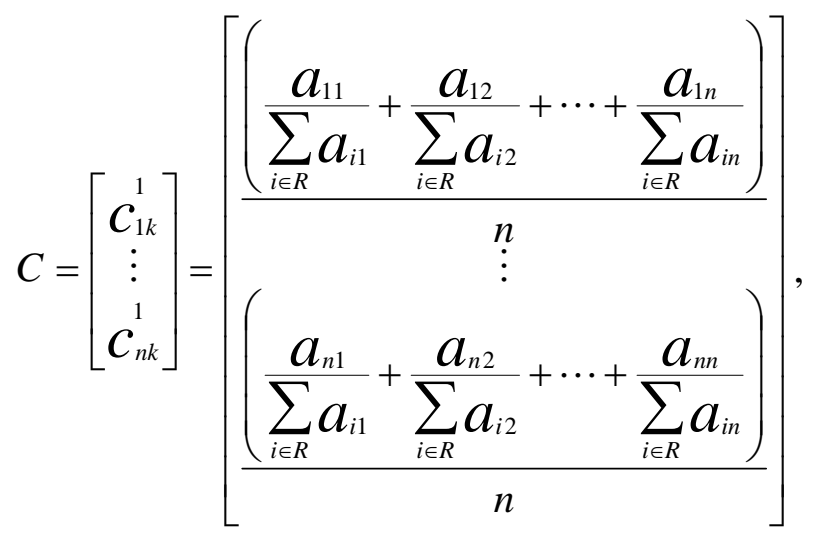

Where $c_{i k}^{1}$ denotes the relationship weightings between the product characteristics $i$ and the corresponding customer requirement $k$.

Step 4: Verify Consistency of AHP

To ensure that the respondent has assigned values from table 1 in a consistent way a consistency test should be carried out. Create a further column matrix by multiplying each entry in column $i$ of matrix $A$ by the column vector $c_{i k}^{1}$ from step 3 then divide by the sum of values in each row $i$ by $c_{i k}^{i}$.

$$
\bar{C}=\left[\begin{array}{c}
-1 \\
C_{1 k} \\
\vdots \\
-1 \\
C_{n k}
\end{array}\right]=\left[\begin{array}{c}
\frac{C_{1 k}^{1} a_{11}+C_{2 k}^{1} a_{12}+\ldots+C_{n k}^{1} a_{1 n}}{C_{1 k}} \\
\vdots \\
\frac{C_{1 k}^{1} a_{n 1}+C_{2 k}^{1} a_{n 2}+\ldots+C_{n k}^{1} a_{n n}}{C_{n k}}
\end{array}\right],
$$

Where $\bar{C}$ is a weighted sum vector. 
Table 1. AHP scale for completing the HoQ comparison matrix.

\begin{tabular}{|c|c|c|}
\hline Intensity & Importance & Explanation \\
\hline 1 & Equal & $\begin{array}{c}\text { Two activities are equally } \\
\text { important }\end{array}$ \\
\hline 3 & Moderate & $\begin{array}{l}\text { One is slightly more } \\
\text { important than the other }\end{array}$ \\
\hline 5 & Strong & $\begin{array}{l}\text { One is strongly more } \\
\text { important than the other }\end{array}$ \\
\hline 7 & Very Strong & One is dominant of the other \\
\hline 9 & Extreme & $\begin{array}{c}\text { Highest possible affirmation } \\
\text { of evidence favoring one } \\
\text { over another. }\end{array}$ \\
\hline $2,4,6,8$ & Intermediate & $\begin{array}{l}\text { Used for compromise when } \\
\text { desired value falls between } \\
\text { above scales }\end{array}$ \\
\hline $\begin{array}{l}\text { Reciprocals of the above } \\
\text { numbers }\end{array}$ & & $\begin{array}{l}\text { Used for inverse } \\
\text { relationships }\end{array}$ \\
\hline
\end{tabular}

Step 5: Calculate the averages of values in vector $\bar{C}$ to give the maximum Eigenvalue $\left(\lambda_{\max }\right)$ of matrix $A$.

$\lambda_{\max }=\frac{\sum_{i \in R}^{-1} C_{i k}}{n}$,

Step 6: Calculate the consistency index.

$C I=\frac{\lambda_{\max }-n}{n-1}$

Step 7: Compute the consistency ratio,

The consistency ratio is based on $\mathrm{RI}(n)$, a random index taken from table 2 based on the value of $n$.

$C R=\frac{C I}{R I(n)}$,

The consistency ratio is a measurement of consistent responses when completing the relationship matrix. If the measurement is greater than 0.10 the process is considered inconsistent and should be repeated in the hope of realizing a more consistent response. This measurement of consistency gives greater confidence when using the AHP-QFD method over the QFD method alone. 
Table 2: List of Random Index values

\begin{tabular}{ccccccccc}
\hline$n$ & 2 & 3 & 4 & 5 & 6 & 7 & 8 & 9 \\
\hline$R I(n)$ & 0 & 0.58 & 0.90 & 1.12 & 1.24 & 1.32 & 1.41 & 1.45 \\
\hline
\end{tabular}

Assuming consistency is acceptable the matrix will be populated with relationship weightings that link the top matrix with the left hand side matrix (Product characteristics with Customer Requirements from Fig. 1). The importance rating of each product characteristic can then be calculated.

Step 8: Compute importance rating

$$
W_{i}^{1}=\sum_{k \in S} p_{k} c_{i k}^{1}
$$

Where $S$ denotes the set of customer requirements $S=\{1,2, \ldots, \mathrm{m}\}$, and $p_{k}$ denotes the importance rating given to that requirement.

The result of step 8 is an importance score for each product characteristic which has been obtained from the requirements of customers. The AHP-QFD method can also be applied to a selection problem. By using the QFD to link the requirements made on a supplier, to the characteristics displayed or possessed by any given supplier, qualitative aspects of supplier selection can be managed in a systematic and robust way.

\subsection{The AHP-QFD approach for bioenergy suppliers}

The QFD method can deal with both qualitative and quantitative aspects of a product or service [7], several other techniques are available for the explicit optimization of fuel blends, therefore the QFD-AHP process will be used to better analyze and understand the qualitative requirements made of suppliers. The method is intended to be applied from a developer perspective as it is the developer that will make the initial decisions on the supply strategy for the scheme. Therefore rather than customer requirements being used as the success criteria the requirements of the developer and the scheme should be identified and weighted.

Many of the requirements of a good biomass supplier are likely to be in line with the requirements on suppliers from other industries. Reliability, company size, responsiveness and quality control are likely to be important tacit requirements. Other requirements may be more unusual and regard the material itself such as accreditation by sustainable forestry bodies, the local or national sourcing of waste, compliance with best practice and the method of delivery may be important aspects not covered by the technical fuel specification.

Having identified the requirements the developer places upon potential suppliers the relative importance of each requirement should be identified. This is done using the first HoQ matrix. The developer is likely to consist of several teams or members of staff with different perspectives on the project. These different teams will place differing importance weightings on different requirements. In this case the developer teams are considered to be equally important, typical developer teams may consist of planning, technical/design teams and finance teams. 


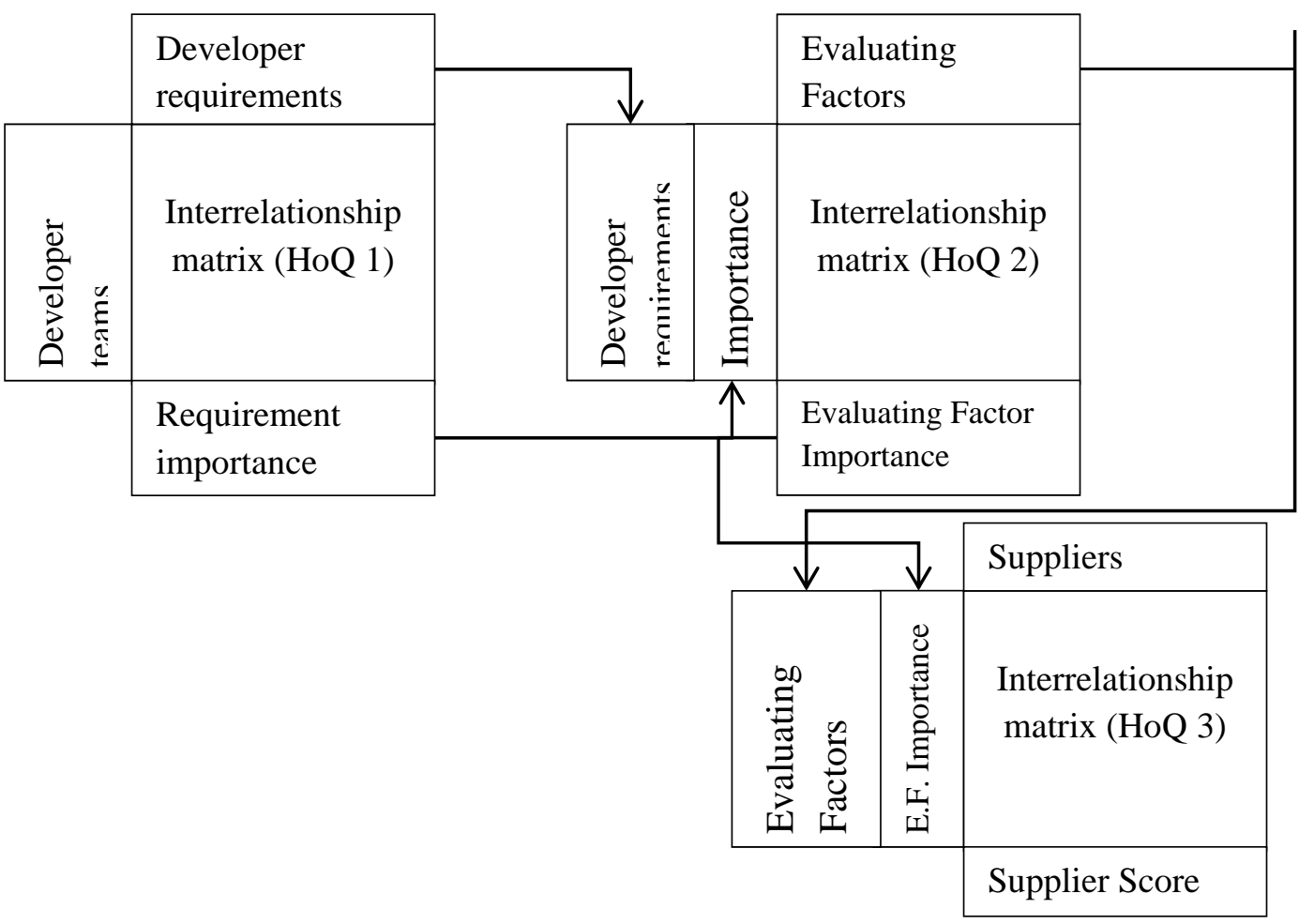

Fig. 2. HoQ 1 giving the importance of each requirement as determined by the developer. HoQ 2 linking the developer requirements with available evaluating factors for potential suppliers and HoQ 3linking those evaluating factors with the available suppliers to the bioenergy scheme.

The output of HoQ 1 is then used in HoQ 2 which links the developer requirements to externally observable characteristics possessed by the suppliers. These characteristics take the place of product characteristics and allow the decision maker to determine to what extent each requirement would be met by certain aspects of a supplier. For instance a requirement for sustainable fuel would be significantly met by a supplier approved by some sustainable forestry stewardship scheme. The use of organic wastes may be negatively related to a requirement to keep site odors to a minimum whilst a guarantee to deliver at within a narrow band of moisture content may score highly against a requirement for consistent fuel characteristics.

A final House of Quality table HoQ 3 can then be constructed which links the evaluating factors and their relative importance with the suppliers available. Here the decision maker must decide to what extent each supplier matches the evaluating factors identified. The output of HoQ 3 is a score for each supplier based on the ability of that supplier to meet the tacit requirements of the developer. Those suppliers that score highest should be favored.

\section{Discussion}

The weighted ranking score of each supplier assists decision makers in determining which suppliers to use when creating a strategy for biomass supply to large scale facilities. The massive quantity of materials required for large scale conversion facilities mean supply chain managers are forced to source from a range of materials and sources. The objective function for optimization algorithms can, using the presented method, incorporate the tacit requirements made on suppliers to create a model constrained by the explicit requirements of material specification imposed by equipment specifications. The objective function therefore could take a form similar that shown in equation (9). 
$\max \left(w_{1} S_{1}+w_{2} S_{2}+\ldots w_{m} S_{m}\right)$

Where $w_{m}$ denotes the score for each supplier $S_{m}$ for $m$ different suppliers. This approach would ensure the resulting suggested strategy is technically feasible, whilst also ensuring the best possible combination of suppliers is contracted.

The AHP-QFD method is suitable for application within developing companies as it is simple to apply and gives predictable and clear outputs for the decision maker. An inherent weakness of the approach is the subjective viewpoint of the decision maker compiling the list of requirements. In this application this weakness is minimized by considering only requirements of the teams within the developer company, not the wider stakeholder group. The AHP-QFD method also has an advantage over other weighting or ranking methods that could be incorporated into the objective function as it directly translates the requirements made on suppliers into their performance score using a robust method rather than a user estimate.

As the research develops more stakeholders could be interviewed for requirements and asked to complete the AHP-QFD process. This would allow developers to gain insight into the requirements that should be satisfied to make the scheme more successful from the perspective of other development stakeholders. As the framework is applied to different stakeholders a database of requirements can be constructed showing global and scheme specific requirements of different stakeholders.

This work is part of a CASE studentship PhD awarded by the ERSC and the authors are grateful for support from Express Energy Holdings UK.

\section{References}

[1] N. Ayoub, E. Elmoshi, H. Seki and Y. Naka, Evolutionary algorithms approach for integrated bioenergy supply chains optimization, Energy Conversion and Management, 50, 2009.

[2] P. Thornley, P. Upham and J. Tomei, Sustainability constraints on UK bioenergy development, Energy Policy, 37, 2009, pp. 5623-5635.

[3] T. Buchholz, E. Rametsteiner, T. Volk and V. Luzadis, Multi Criteria Analysis for bioenergy systems assessments, Energy Policy, 37, 2009, pp. 484-495.

[4] W. Ho, An integrated analytical approach for selecting suppliers strategically, 3rd World Conference on Production and Operations Management, 2008, CD-ROM.

[5] N. Hanumaiah, B. Ravi and N. P. Mukherjee, Rapid hard tooling process selection using QFD-AHP methodology, Journal of Manufacturing Technology Management, 17, 2006, pp. 332 - 350.

[6] S. Myint, A framework of an intelligent quality function deployment (IQFD) for discrete assembly environment, Computers \& Industrial Engineering, 45, 2003, pp. 269-283.

[7] C. N. Madu, C. Kuei and I. E. Madu, A hierarchic metric approach for integration of green issues in manufacturing: a paper recycling application, Journal of Environmental Management, 64, 2002, pp. 261-272. 